\title{
Comportamiento de la vegetación a partir del índice verde y datos climáticos en dos sitios de la Patagonia Austral
}

\section{Behavior of the vegetation from the green index and climate data in two sites of the Austral Patagonia}

\author{
Julio Soto $^{1}$, Paula Paredes ${ }^{1,2}$, Dora Maglione ${ }^{1}$, Marisa Sandoval ${ }^{1}$, Oscar Bonfili ${ }^{3}$, \\ jsoto@uarg.unpa.edu.ar,pparedes@uarg.unpa.edu.ar,dmaglione@uarg.unpa.edu.ar, \\ msandoval@uarg.unpa.edu.ar,oscar.bonfili@gmail.com \\ ${ }^{1}$ Universidad Nacional de la Patagonia Austral, Unidad Académica Río Gallegos - Instituto \\ ITET e ICASUR \\ ${ }^{2}$ INTA - EEA Santa Cruz, Grupo RRNN \\ ${ }^{3}$ Servicio Meteorológico Nacional, Oficina Meteorológica Río Gallegos
}

Recibido: 28/05/2020. Aceptado: 14/12/2020

\section{RESUMEN}

La cantidad de humedad al sur de la Patagonia (argentino-chilena), en parte condicionada por la presencia de la cordillera, determina cambios en los atributos de las comunidades vegetales. Los índices de vegetación obtenidos por sensores remotos están asociado a la cantidad de material fotosintéticamente activo y Productividad Primaria Neta Aérea (PPNA), entre otras características de la vegetación. El índice de vegetación más utilizado es el índice Verde Diferencia Normalizada (NDVI). Particularmente la I-NDVI (Integral del índice de vegetación) está asociada a PPNA. El objetivo de este trabajo es describir el comportamiento de la vegetación en dos sitios del sur de la Patagonia (Río Gallegos y Punta Arenas) a través del NDVI y su relación con variables meteorológicas. Datos de precipitación y temperatura fueron obtenidos de estaciones meteorológicas y los datos espectrales (NDVI- de imágenes MOD13Q1, 250m, 16 días) utilizando la plataforma Google Earth Engine. Se calcularon índices de precipitación estandarizados (SPI y SPEI). Se realizaron correlaciones entre los datos meteorológicos y espectrales. El NDVI describió el comportamiento de la vegetación. La evolución intraanual del NDVI es diferente en los sitios evaluados. El área de Punta Arenas presenta valores más altos todo el año (mayor I-NDVI) y un comportamiento unimodal (máximo en diciembre), mientras que Río Gallegos presentó dos picos (octubre y abril). Resultados preliminares indican que el NDVI está correlacionado con temperatura anual en Punta Arenas, mientras que en Río Gallegos correlacionó con la precipitación acumulada. La I-NDVI estuvo correlacionado con SPI y SPEI (10 meses) solo en Río Gallegos.

Palabras clave: índice NDVI; variables climatológicas; índices de precipitación.

\begin{abstract}
The amount of moisture south of Patagonia (Argentine-Chilean), partly conditioned by the presence of the mountain range, determines changes in the attributes of plant communities. Vegetation indices obtained by remote sensors are associated with the amount of photosynthetically active material and Annual Net Primary Productivity (ANPP), among
\end{abstract}


other characteristics of the vegetation. The most used vegetation index is the Normalized Difference Vegetation Index (NDVI). Particularly the I-NDVI (Integral of the vegetation index) is associated with ANPP. The objective of this work is to describe the behavior of vegetation in two sites in southern Patagonia (Río Gallegos and Punta Arenas) through the NDVI and its relationship with meteorological variables. Precipitation and temperature data were obtained from meteorological stations and spectral data (NDVI- of MOD13Q1 images, 250m, 16 days) using the Google Earth Engine platform. Standardized precipitation indices (SPI and SPEI) were calculated. Correlations were made between meteorological and spectral data. The NDVI described the behavior of the vegetation. The intra-annual evolution of NDVI is different in the sites evaluated. The Punta Arenas area has higher values throughout the year (higher I-NDVI) and unimodal behavior (maximum in December), while Río Gallegos presented two peaks (October and April). Preliminary results indicate that the NDVI is correlated with annual temperature in Punta Arenas, while in Río Gallegos it correlated with the accumulated rainfall. The I-NDVI was correlated with SPI and SPEI (10 months) only in Río Gallegos.

Keywords: NDVI index; weather variables; precipitation rates.

\section{INTRODUCCIÓN}

El clima al sur de la Patagonia Austral (argentino- chilena) está asociado a la dinámica de los procesos meteorológicos en contacto con los factores geográficos y estacionales que caracterizan a la Patagonia en general, y a la ciudad de Río Gallegos (Argentina) y Punta Arenas (Chile) en particular. Estas dos ciudades se encuentran en medio de la gran corriente austral del oeste, lo que permite una circulación casi permanente de vientos intensos en esa dirección. Considerando el régimen hídrico, el lado chileno es húmedo-subhúmedo, y el argentino semiárido, y en cuanto al régimen de temperaturas, el primero se clasifica como oceánico y el segundo como de transición (Coronato, Mazzoni, Vázquez y Coronato, 2017). La cantidad de humedad al sur de la Patagonia, en parte, condicionada por la presencia de la cordillera, determina cambios en los atributos de las comunidades vegetales, de un lado y otro de este orógeno. Tales atributos, como biomasa, diversidad, formas de vida, Productividad Primaria Neta Aérea (PPNA) son relevantes para cada ecosistema en particular.

La PPNA, cantidad de biomasa vegetal generada en un tiempo determinado, es una variable de importancia para el funcionamiento de los ecosistemas, porque es la base de la cadena trófica (Odum, 1971), regulando así el flujo de energía (McNaughton, Oesterheld, Frank y Williams, 1989). La PPNA también representa un estimador de la cantidad de servicios ecosistémicos que ofrecen los sistemas naturales (Costanza et al., 1998).

La PPNA es variable tanto en el tiempo como en el espacio a diferentes escalas. Poder conocer esta variación es de importancia cuando se pretende manejar los sistemas naturales, principalmente los ganaderos, ya que es el principal determinante de la carga animal que puede sustentar (Oesterheld, Di Bella y Kerdiles, 1998). La PPNA de los pastizales, y su variación en el tiempo, se encuentran íntimamente ligadas a la cantidad y distribución de las precipitaciones anuales (Lauenroth, 1979; Sala, Parton, Joyce y Lauenroth, 1988), con la estación del año (variación intraanual) y puede encontrarse asociación con eventos climáticos si se analiza como una serie de tiempo. La precipitación, junto con otros elementos del clima determinan la distribución regional de tipos de pastizales, la composición de especies (Odum, 1971), las formas de vida de la vegetación y la variación interanual de la PPNA (Hill, Donald, Hyder y Smith, 2004; Sala, Lauenroth y Golluscio, 1997; Solbrig et al., 1977). La distribución espacial de la PPNA, se relaciona también con otros factores ambientales, como el tipo de 
suelo y cambios en su uso (Volante, Alcaraz-Segura, Mosciaro, Viglizzo y Paruelo, 2012; Baldi, Verón y Jobbágy, 2013), humedad ambiente, vientos predominantes, etc.

Es posible cuantificar la PPNA a través de diferentes métodos, estos se clasifican en directos e indirectos. El método directo consiste en cortes sucesivos de biomasa (Sala y Austin, 2000), lo cual requiere gran tiempo, dinero y la información obtenida es puntual, por lo que extrapolar los resultados genera imprecisión. El método indirecto consiste en la estimación de la PPNA a partir de datos obtenidos de sensores remotos (Paruelo et al., 2000; Paruelo y Lauenroth, 1998; Pickup, Chewings y Nelson, 1993), con previo ajuste a cada región, y presenta la ventaja de poder obtener la información para grandes áreas de manera rápida, económica y con mayor frecuencia.

En diferentes trabajos se ha encontrado una relación positiva entre índices espectrales de vegetación y los distintos atributos. Los índices de vegetación, obtenidos por sensores remotos, están asociado a la cantidad de material fotosintéticamente activo, por lo tanto, con PPNA, cobertura vegetal y evapotranspiración (Di Bella, Rebella y Paruelo, 2000; Fabricante, 2005; Goward, Tucker y Dye, 1985; Irisarri, 2008; Paruelo, Epstein, Lauenroth y Burke, 1997) entre otras características de la vegetación. Existen diferentes índices de vegetación (Gilabert, González-Piqueras, García-Haro, 1997; Paredes, 2011; Wan, 1999), que consideran el comportamiento de la reflectancia en diferentes longitudes de onda. El índice de vegetación más utilizado es el índice Verde Diferencia Normalizada (NDVI- Normalized Difference Vegetation Index)(Rouse, Haas, Schell y Deering, 1973), que se calcula como el cociente entre las bandas del Infrarrojo Cercano (NIR) y la del Rojo (R):

$$
\mathrm{NDVI}=(\mathrm{NIR}-\mathrm{R}) /(\mathrm{NIR}+\mathrm{R})
$$

(ecuación 1)

donde NIR, en imágenes MODIS (Mod13Q1), está en la longitud de onda $(\lambda)$ de $858 \mathrm{~nm}$ y la banda del Rojo en $645 \mathrm{~nm}$.

Otro índice de vegetación, EVI (Enhanced Vegetation Index) (Huete et al., 2002), incorpora la reflectancia en la zona del azul del espectro visible, por lo que el efecto del suelo se ve disminuido. Se calcula como:

$$
\mathrm{EVI}=[2,5(\mathrm{NIR}-\mathrm{R})] /(\mathrm{NIR}+\mathrm{C} 1 * \mathrm{C} 2 * \mathrm{Azul}+l)
$$

\section{(ecuación 2)}

donde Azul, corresponde en MODIS, a $469 \mathrm{~nm}, l=$ factor de corrección del brillo del suelo; C1 y C2 son coeficientes de correlación de aerosoles. El valor utilizado para el parámetro $l$ fue de 0,5 (recomendado en bibliografía para sitios con nivel de canopeo intermedio) y los coeficientes $\mathrm{C} 1=6, \mathrm{C} 2=7,5$ y $l=1$.

Existen índices espectrales relacionado al contenido hídrico de las plantas y el suelo. El Índice Diferencial de Agua Normalizado (NDWI) (Hunt y Rock, 1989, Gao, 1996), refleja el contenido de humedad de la vegetación y del suelo y se determina combinando la franja del NIR y el Infrarrojo Medio (MIR):

$$
\mathrm{NDWI}=(\mathrm{NIR}-\mathrm{MIR}) /(\mathrm{NIR}+\mathrm{MIR})
$$

(ecuación 3)

donde MIR corresponde a $\lambda=(2130 \mathrm{~nm} / 2105-2155 \mathrm{~nm})$ en MOD13Q1.

Paruelo, Jobbágy y Sala (1998) establecen que calculando tres atributos de la curva estacional del NDVI, y considerando que éste está relacionado con la PPNA, se puede determinar el comportamiento funcional del ecosistema. Estos atributos son: Integral anual (I-NDVI), Rango Relativo anual (RRel), el valor y Momento del Máximo (MMax). Particularmente la INDVI (Integral del índice de vegetación) está asociada a la cantidad de PPNA, mientras que 
el RRel está relacionado a la variabilidad del NDVI dentro del año y el MMax al momento de máxima acumulación de biomasa verde.

La estación de crecimiento (inicio y finalización), así como su longitud, son variables que describen el comportamiento fenológico de determinado tipo de vegetación y están íntimamente relacionadas al tipo de clima (Jobbágy, Sala y Paruelo, 2002). La curva de evolución anual del NDVI permite obtener estos parámetros. Existen diferentes metodologías para determinar el inicio y finalización de la estación de crecimiento (Jobbágy et al, 2002; Jönsson y Eklundh, 2006; Kaduk y Heimann, 1996; Reed et al., 1994; White, Thornton y Running, 1997).

Existen varios índices (espectrales o climáticos) que cuantifican algún tipo de sequía. Dentro de los espectrales, por ejemplo, si se analiza la tendencia del NDVI en una serie de tiempo puede encontrarse asociación con estos eventos climáticos (Farías, Dessí, Maselli y Crotti, 2005; Alatorre, Beguería y Vicente-Serrano, 2010; Gu, Brown, Verdin, y Wardlow, 2007; Ji y Peters, 2003). Dentro de los índices climáticos, los más utilizados son: el Índice Estandarizado de Precipitación (SPI), asociado a sequías meteorológicas, y el Índice Estandarizado de Precipitación Evapotranspiración (SPEI), más asociado a sequías agrícolas. El SPI (McKee, Doesken y Kleist, 1993 y 1995) es un índice potente y flexible; se concibió para cuantificar el déficit/exceso de precipitación para múltiples escalas temporales o "ventanas de promedios. El índice SPEI, que es una variación del índice anterior, elaborada por Vicente-Serrano, Beguería y López-Moreno (2010), incorpora el efecto de la evapotranspiración que influye en las condiciones de sequía. Ambos índices pueden ser calculados en distintas escalas temporales. La aplicación más práctica varía entre 1 y 24 meses (Guttman, 1994, 1999), aunque mientras más grandes sean las escalas, mayor debería ser la longitud de la serie para conservar la confiabilidad de los resultados.

El objetivo principal de este trabajo es describir el comportamiento de la vegetación en dos sitios del sur de la Patagonia: Sitio 1 (Río Gallegos - Argentina) y Sitio 2 (Punta Arenas Chile) a través del NDVI y su relación con variables meteorológicas.

\section{MATERIALES Y MÉTODOS}

El área de estudio comprendió dos sitios: Sitio 1, área aledaña a Río Gallegos (Argentina) y Sitio 2, al norte de Punta Arenas (Chile). Río Gallegos posee un clima Seco Semiárido en donde la evapotranspiración excede la precipitación media anual, las precipitaciones varían de 200 - $300 \mathrm{~mm}$ y el promedio anual de temperaturas está entre 6 y $7^{\circ} \mathrm{C}$. Este sitio se encuentra dentro del área ecológica Estepa Magallánica Seca, caracterizada por el crecimiento de una vegetación dominada por pastizales de coirón fueguino (Festuca gracillima), con coberturas variables (60-70\%) (Oliva, González, Rial y Livraghi, 2001). En el Sitio 2 (Punta Arenas), el tipo de clima que predomina es el Templado húmedo lluvioso, sin estación seca en todo el año con veranos cortos y frescos (según Köppen-Geiger), se ubicada dentro del distrito agroclimático homónimo, posee mayores precipitaciones anuales $(432 \mathrm{~mm})$ con temperatura media anual de $6,5^{\circ} \mathrm{C}$ y con una amplitud térmica menor a Río Gallegos, con una vegetación dominada por praderas naturales de Festuca gracillima y Festuca pallescens, Vegas y comunidades de Murtilla (Empetrum rubrum) (Endlicher y Santana, 1988).

Los datos de precipitación, temperatura y velocidad del viento fueron obtenidos de las estaciones meteorológicas de cada localidad (estación Río Gallegos del Servicio Meteorológico Nacional https://www.smn.gob.ar/ y la estación de Punta Arenas de la Dirección Meteorológica de Chile http://www.meteochile.gob.cl/PortalDMCweb/index.xhtml). Las series temporales analizadas corresponden a datos mensuales entre enero de 1964 y diciembre de 2015. A partir de estas series se calcularon índices de 
precipitación estandarizados (SPI y SPEI), para diferentes escalas temporales. También se realizó el cálculo de Balance Hídrico (BAL) y de la Evapotranspiración Potencial (PET), utilizando para esta última la ecuación de Thornthwaite (1948).

El cálculo del SPI sólo requiere de la precipitación observada, la cual fue ajustada a una distribución de probabilidades del tipo gamma, y luego se estandarizó a una normal estándar para definir distintas intensidades de la sequía. Para el cálculo del SPEI se utilizaron las series de precipitaciones, temperaturas y la latitud de cada localización usando para el ajuste una distribución teórica log-logística. Para este trabajo se utilizó una escala temporal de 1, 3, 6, 9 y 12 meses.

Los datos espectrales (NDVI, EVI, banda 7 y 2) se extrajeron de imágenes MODIS (producto MOD13Q1, resolución espacial $250 \mathrm{~m}$, y compuesto de 16 días) utilizando la plataforma Google Earth Engine. Se generaron áreas de interés (polígonos) en zonas adyacentes a las estaciones meteorológicas (Mapa 1) para extraer la información promedio por fecha (desde el año 2000 al 2015) para cada polígono. Con los datos de la banda 7 (MIR) y 2 (NIR) se calculó el índice de contenido hídrico (NDWI).

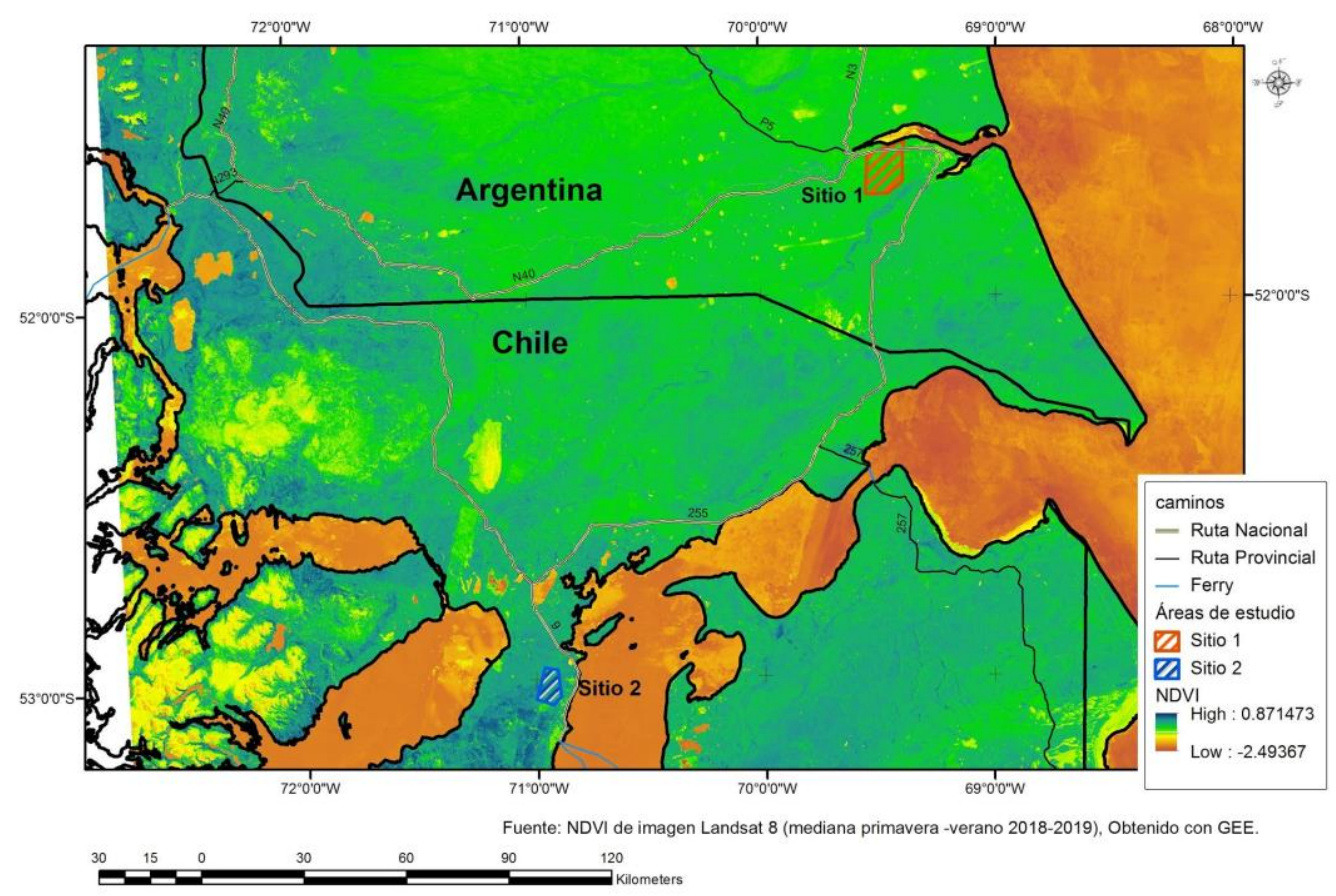

Mapa 1: Ubicación de los polígonos de los cuales se extrajo información desde la plataforma GEE. El polígono rojo corresponde al Sitio 1 (área de Río Gallegos), y el azul, al Sitio 2 (área de Punta Arenas.

Se calcularon los atributos de la curva de NDVI. La Integral anual (I-NDVI) de cada sitio se calculó como el promedio anual, no considerando los meses de Junio y Julio, debido a la interferencia de la señal que puede provocar la presencia de nieve y alta nubosidad en esos meses. Para calcular el inicio y finalización de la estación de crecimiento se consideró el momento en el cual la curva de evolución anual del NDVI se cruza con el promedio anual. El momento del máximo es el momento (mes) en el cual el valor anual es máximo.

Luego de la comprobación de la homogeneidad de las series temporales de datos a fin de detectar posibles errores en los mismos, se realizaron correlaciones (mensuales y generales) entre los datos meteorológicos, índices espectrales e índices estandarizados de precipitación, para cada localidad. También se realizaron correlaciones entre NDVI y las precipitaciones de 
los tres meses anteriores. Se elaboraron diferentes modelos de regresión para el NDVI, teniendo en cuenta algunas de las variables bajo estudio.

Todos los datos fueron analizados estadísticamente utilizando los software Infostat (Di Rienzo et al, versión 2018) y R (R Core Team, 2018).

\section{RESULTADOS}

El NDVI describió el comportamiento de la vegetación. La evolución interanual del índice de vegetación presentó, para todos los años analizados, valores más altos en el Sitio 2 (Figura 1, 2 y Tabla 1). La evolución intraanual del NDVI también fue diferente en los sitios evaluados. El área del Sitio 2 (Punta Arenas - Chile) presenta valores más altos todo el año (mayor INDVI), una mayor dispersión y un comportamiento unimodal (Momento del máximo en diciembre), mientras que la zona del Sitio 1 (Río Gallegos - Argentina), presentó dos picos (Momento del máximo en octubre y un pico de menor magnitud en abril), y menor dispersión de los datos (Figura 1, 2 y Tabla 1).

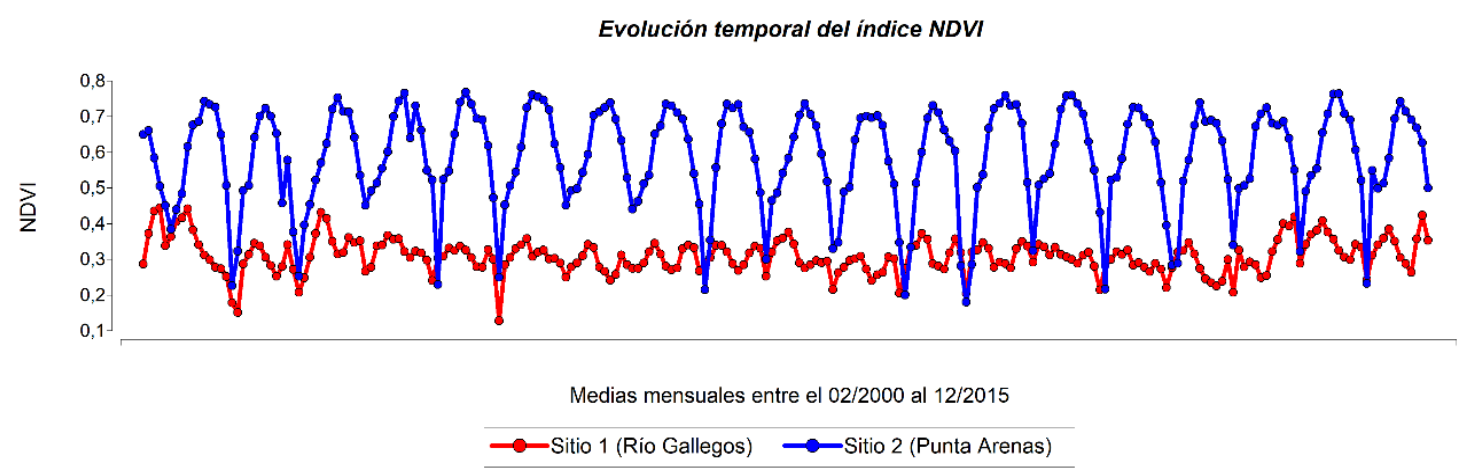

Figura 1: Serie (2000-2015) del NDVI mensual para los sitios estudiados.

Las correlaciones entre los índices de vegetación (NDVI y EVI), para cada localidad, fue alta. Para todos los datos mensuales del periodo considerado, se observa que existe una correlación alta entre ambos (Sitio $1 \mathrm{r}=0,76$, Sitio $2 \mathrm{r}=0,95$ ). Si se calculan para cada mes la correlación también es alta: en el Sitio 1, con valores que van desde los 0,72 a 0,89 (dependiendo del mes), excepto para septiembre y diciembre en que es muy alta ( $r=0,90$ y $r=0,96$, respectivamente); mientras que en el Sitio 2, los meses de marzo, junio, julio, agosto y noviembre la correlación es muy alta y es alta en los meses restantes. Debido a esta alta correlación entre índices se decidió trabajar sólo con NDVI, que es además el índice más ampliamente utilizado en la bibliografía.

Considerando los datos climáticos de la serie analizada se encontraron diversas correlaciones. Las correlaciones entre las precipitaciones de ambas ciudades fueron moderadas $(r=0,45)$. La temperatura promedio correlacionó positiva y significativamente con PET (Sitio $1 \mathrm{r}=0,98$, Sitio $2 \mathrm{r}=0,97$ ), mientras que la precipitación correlacionó negativamente con BAL (Sitio 1 $r=-0,86$, Sitio $2 r=-0,80)$. 


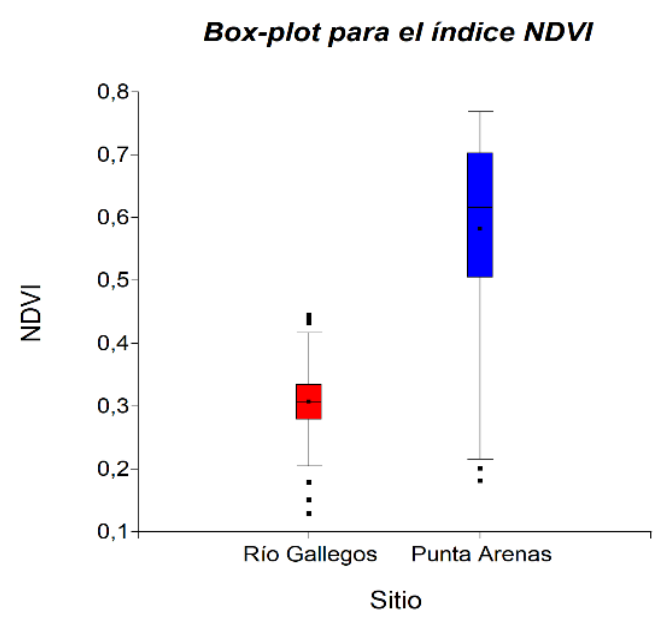

Figura 2: Boxplot de los datos de NDVI, para la serie analizada, de cada sitio.
Tabla 1: Medidas resúmenes de NDVI para cada sitio

\begin{tabular}{|l|l|l|}
\hline NDVI & $\begin{array}{l}\text { SITIO 1 } \\
\text { Río } \\
\text { Gallegos }\end{array}$ & $\begin{array}{l}\text { SITIO 2 } \\
\text { Punta } \\
\text { Arenas }\end{array}$ \\
\hline Media & $\mathbf{0 , 3 1}$ & $\mathbf{0 , 5 9}$ \\
D.E. & $\mathbf{0 , 0 5}$ & $\mathbf{0 , 1 4}$ \\
CV & $\mathbf{1 5 , 6 5}$ & $\mathbf{2 3 , 8 3}$ \\
Mínimo & $\mathbf{0 , 1 3}$ & $\mathbf{0 , 1 8}$ \\
Máximo & $\mathbf{0 , 4 4}$ & $\mathbf{0 , 7 7}$ \\
Mediana & $\mathbf{0 , 3 1}$ & $\mathbf{0 , 6 2}$ \\
Q1 & $\mathbf{0 , 2 8}$ & $\mathbf{0 , 5 1}$ \\
Q3 & $\mathbf{0 , 3 4}$ & $\mathbf{0 , 7 0}$ \\
\hline
\end{tabular}

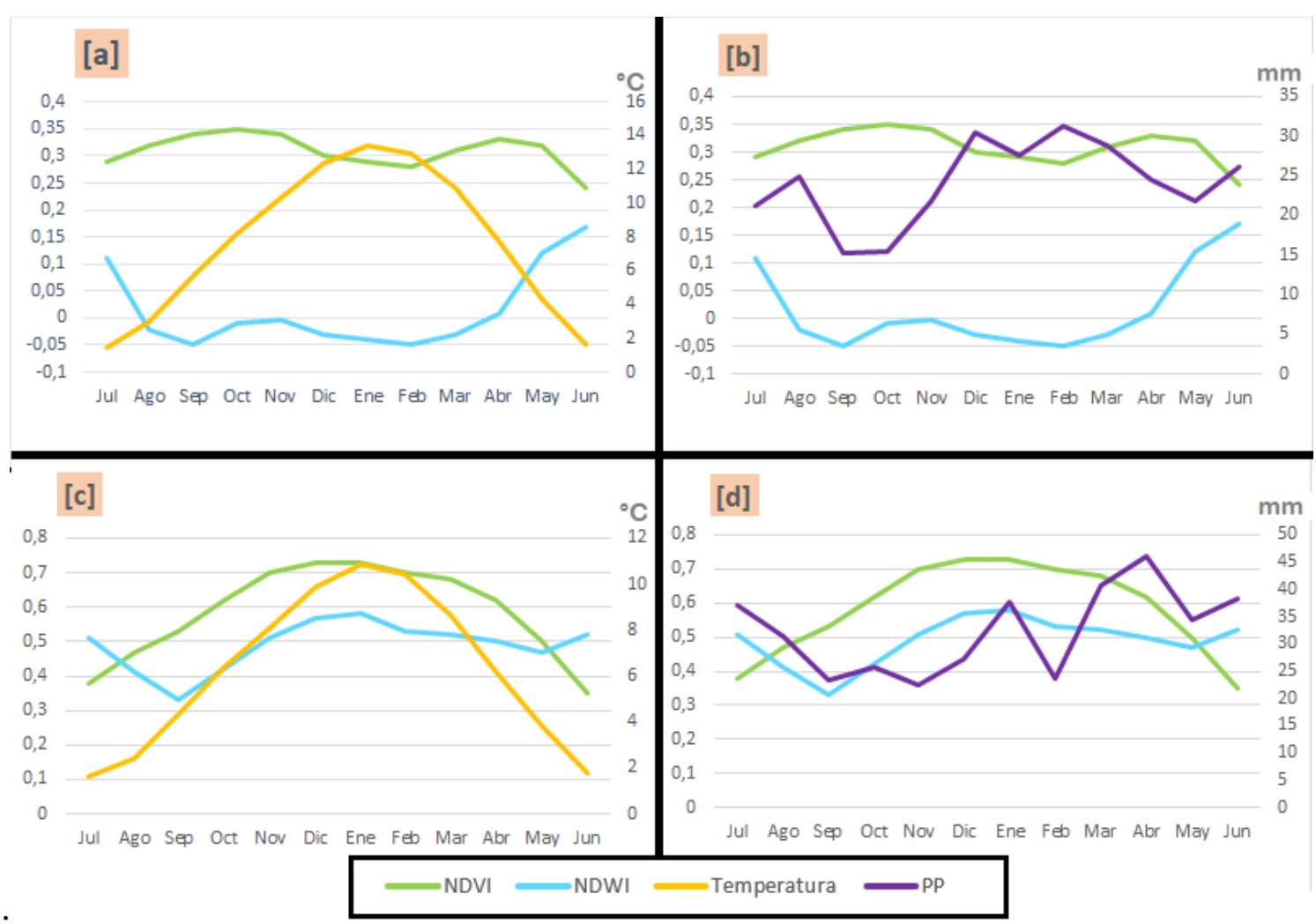

Figura 3: Evolución de valores medias mensuales para las variables analizadas: NDVI, NDWI, Temperatura $\left(e n{ }^{\circ} \mathrm{C}\right)$ y Precipitación (en mm), para el Sitio 1 (a y b) y el Sitio 2 (c y d).

El índice de Humedad (NDWI) mostró un comportamiento anual vinculado a la precipitación y a la temperatura (Figura 3). En el Sitio 1, en verano se observan las principales precipitaciones, y las temperaturas más altas, ocasionando que la humedad de la vegetación y el suelo disminuya (mayor PET y por lo tanto menor NDWI). En el invierno, momento de menores temperaturas, el contenido hídrico es mayor. Sin embargo, el Sitio 2, al tener precipitaciones abundantes a lo largo del año, y temperaturas relativamente más bajas que el Sitio 1, tiene mayor NDWI. 
La estación de crecimiento fue distinta en ambos Sitios (Figura 4). En el Sitio 1 hay dos períodos de crecimiento: el primero inicia en agosto y finaliza a fines de noviembre (aproximadamente 151 días, siendo el momento del máximo en octubre), y el segundo de menor magnitud inicia a mediados de marzo y finaliza a principios de mayo (aproximadamente 76 días con el momento del máximo en abril). Mientras que en el Sitio 2 hay un solo periodo de crecimiento, que inicia a mediados de septiembre y finaliza a mediados de abril, con momento de máximo en diciembre, siendo la duración de la estación de crecimiento de aproximadamente 213 días.

\section{Media mensual del índice NDVI}

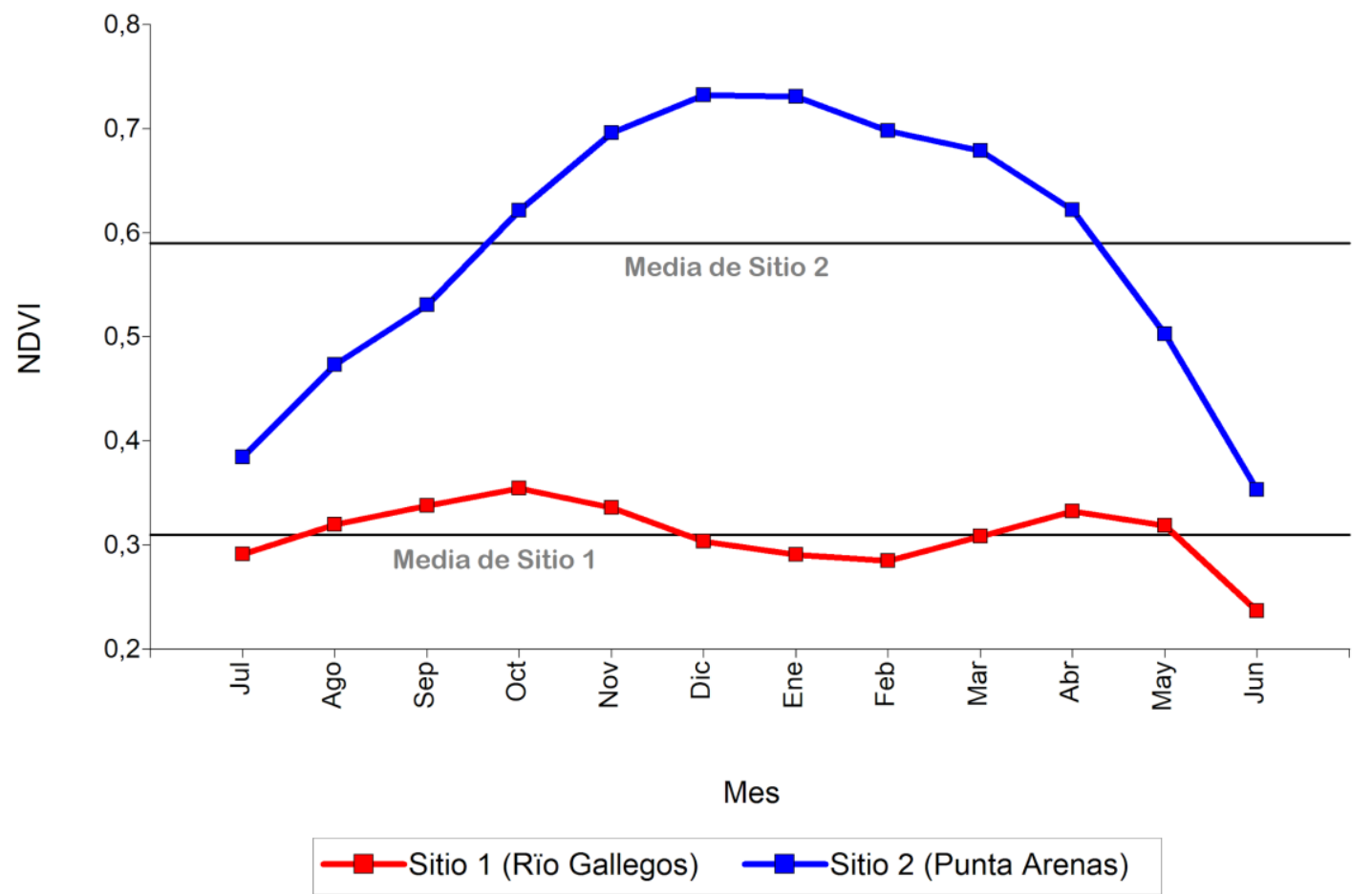

Figura 4: Media mensual de NDVI y, media (gris), para cada sitio.

Los Índices de Precipitación analizados (SPI y SPEI) resultaron correlacionados en cada localidad siendo muy altas y positivas (Sitio 1 entre 0,92 y 0,93 y en Sitio 2 entre 0,93 y 0,95) para todas las escalas temporales consideradas.

El NDVI resultó correlacionado positiva y moderadamente con el SPI calculado para 6 y 9 meses en Río Gallegos, mientras que en Punta Arenas las correlaciones con los índices de sequía, calculados en todas las escalas temporales fueron bajas o muy bajas. Considerando una escala anual, los períodos húmedos y secos, para Río Gallegos, se observan también a través del NDVI. En el Sitio 1 la integral del NDVI (I-NDVI) presenta una correlación positiva moderada con los índices SPI y SPEI (escala temporal anual) ( $r=0,52$ y $r=0,54$ respectivamente).

En función de los periodos de crecimiento que se observan en el Sitio 1, se consideraron tres periodos a analizar:
A) Diciembre-Enero (Máxima acumulación de biomasa- floración)
B) Septiembre-Octubre-Noviembre (máxima tasa de crecimiento)
C) Marzo-Abril (rebrote de otoño).

Se modeló al NDVI, para cada Sitio, en función de los períodos considerados, utilizando las variables: Temperatura Media (TM), Balance Hídrico (BAL), Precipitación del mes (PP) y 
Precipitaciones de los tres meses anteriores (PP_1, PP_2, PP_3) usando una estructura autorregresiva de orden 1 para los errores (Modelos 1 y 2 y Tablas 2 y 3). Para el Sitio 1 no se consideró la PET ya que la correlación con la temperatura es muy alta $(\mathrm{r}=0,9)$ y para el Sitio 2 no se consideraron ni PET ni BAL por poseer correlaciones altas con temperatura y precipitación respectivamente. Los modelos ajustados fueron:

- en el Sitio 1 (Modelo 1):

NDVI $=\mathbf{a}_{0}+\mathbf{a}_{1} \mathbf{T M}+\mathbf{a}_{2} \mathbf{B A L}+\mathbf{a}_{3} \mathbf{P P}+\mathbf{a}_{4} \mathbf{P P} \_1+\mathbf{a}_{5} \mathbf{P P} \_\mathbf{2}+\mathbf{a}_{6} \mathbf{P P} \_3+\mathbf{e}$ con e $\sim \mathrm{AR}:(1)$

- en el Sitio 2 (Modelo 2):

$\mathbf{N D V I}=\mathbf{a}_{0}+\mathbf{a}_{1} \mathbf{T M}+\mathbf{a}_{3} \mathbf{P P}+\mathbf{a}_{4} \mathbf{P P} \_1+\mathbf{a}_{5} \mathbf{P P} \_2+\mathbf{a}_{6} \mathbf{P P} \_3+\mathbf{e}$ con e $\sim \mathrm{AR}(1)$.

Para el Sitio 1 (Río Gallegos), con una confianza del 95\%, se puede concluir que las precipitaciones de los tres meses anteriores, temperatura y BAL influyen sobre el índice NDVI. Además, existen diferencias entre los valores medios de NDVI en los periodos considerados, siendo septiembre-octubre-noviembre el que presenta los mayores valores de NDVI y que es significativamente diferente a los otros dos (Tabla 2).

Modelando los períodos en forma independiente, para Río Gallegos, se observa que en septiembre-octubre-noviembre la variable que influye sobre el NDVI es la precipitación del mes y la del mes previo, en diciembre-enero además de las anteriores influyen la temperatura y BAL, y en durante marzo-abril todas las variables influyen (precipitación del mes y de los tres anteriores, temperatura y balance hídrico) (Tabla 3).

Tabla 2: p-valores para para las pruebas de significación de los parámetros para el modelo considerado en cada sitio

\begin{tabular}{|l|l|l|} 
& $\begin{array}{l}\text { RIO GALLEGOS } \\
\text { p-valor }\end{array}$ & $\begin{array}{l}\text { PUNTA ARENAS } \\
\text { p-valor }\end{array}$ \\
\hline Intercepto & $<\mathbf{0 , 0 0 0 1}$ & $<\mathbf{0 , 0 0 0 1}$ \\
Diferencia período A con B & $\mathbf{0 , 0 0 0 1}$ & $\mathbf{0 , 0 1 6 2}$ \\
Diferencia período A con C & 0,6342 & 0,2393 \\
PP del mes anterior & $\mathbf{0 , 0 0 1 1}$ & 0,8188 \\
PP de dos meses anteriores & $\mathbf{0 , 0 3 9 8}$ & 0,0811 \\
PP de tres meses anteriores & $\mathbf{0 , 0 2 0 0}$ & 0,4787 \\
Precipitación del mes en curso & 0,0681 & 0,1363 \\
Temperatura & $\mathbf{0 , 0 0 3 8}$ & $<\mathbf{0 , 0 0 0 1}$ \\
Balance hídrico & $\mathbf{0 , 0 1 2 0}$ & ----- \\
\hline
\end{tabular}

Tabla 3: p-valores para las pruebas de significación de los parámetros del modelo en cada periodo considerado en el sitio Río Gallegos

\begin{tabular}{|l|l|l|l|}
\hline Variable (p-valor) & Sep-Oct-Nov & Dic-Ene & Mar-Abr \\
\hline PP & $\mathbf{0 , 0 3 7 5}$ & $\mathbf{0 , 0 0 0 1}$ & $\mathbf{0 , 0 0 2 0}$ \\
PP del mes anterior & $\mathbf{0 , 0 2 6 1}$ & $\mathbf{0 , 0 0 0 4}$ & $<\mathbf{0 , 0 0 0 1}$ \\
PP de dos meses & 0,4577 & 0,0844 & $<\mathbf{0 , 0 0 0 1}$ \\
anteriores de tres meses & 0,4750 & 0,9152 & $\mathbf{0 , 0 0 9 8}$ \\
PP de & 0,0763 & $<\mathbf{0 , 0 0 0 1}$ & $\mathbf{0 , 0 0 1 0}$ \\
anteriores & 0,0620 & $\mathbf{0 , 0 0 0 1}$ & $\mathbf{0 , 0 0 2 0}$ \\
Temperatura & & & \\
Balance Hídrico & & & \\
\hline
\end{tabular}


En el Sitio 2 (Punta Arenas) el valor de NDVI está influenciado por la temperatura (pvalor $<0,0001$ ), y además hay diferencia entre los valores medios de NDVI en los períodos, siendo los periodos diciembre-enero y septiembre-octubre-noviembre estadísticamente diferentes (Tabla 3). Para los períodos septiembre-octubre-noviembre y marzo-abril la variable que influye es la temperatura ( $\mathrm{p}$-valor $<0,0001)$.

La velocidad del viento en ambos Sitios presenta el mismo patrón, aunque es mayor en Río Gallegos durante todo el año (Figura 5), con una correlación de 0,89 y un máximo en Noviembre. La velocidad de los vientos resultó correlacionada con las otras variables atmosféricas y algunos de los índices espectrales. En Río Gallegos se encontraron correlaciones muy altas con PET $(0,93)$, BAL $(-0,94)$, altas con temperatura $(0,88)$, EVI $(0,78)$, NDWI $(-0,65)$ y baja con NDVI $(0,21)$. Mientras que en Punta Arenas fueron altas con BAL $(-0,83)$, PET $(0,78)$, Temperatura $(0,72)$, EVI $(0,78)$, NDVI $(0,78)$ y casi nula con NDWI $(0,06)$.

\section{Media mensual de la Velocidad del Viento}

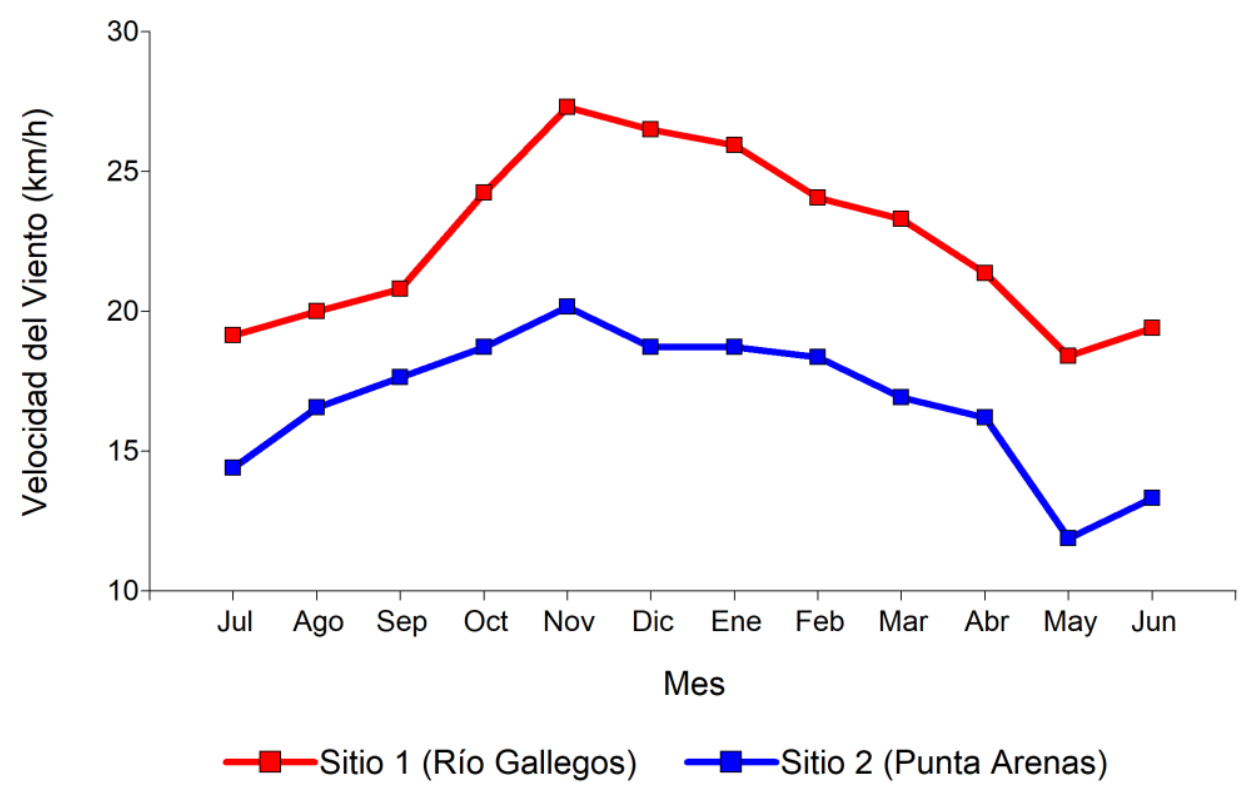

Figura 5. Velocidad promedio mensual para los sitios estudiados.

\section{DISCUSION Y CONCLUSIONES}

El NDVI describe el comportamiento de la vegetación dentro del año, permitiendo establecer además el inicio y finalización de la estación de crecimiento para ambos Sitios. En el Sitio 1 (Río Gallegos) el índice de vegetación muestra dos estaciones de crecimiento, la más importante de Agosto hasta fines de Noviembre, y uno pequeño de mediados de Marzo hasta principios de Mayo con una duración aproximada de 151 y de 76 días respectivamente (227 días en total). Según Borrelli (1998) el mayor crecimiento de la vegetación para la zona de la Estepa Magallánica Seca se produce posterior a la estación invernal, siendo la máxima acumulación de biomasa al final de la primavera, aunque puede variar de año a año y en función de la posición topográfica (Ferrante, 2011). Paredes (2011), utilizando datos de NDVI, encontró para la Estepa Magallánica Seca, resultados similares a los del presente trabajo, con un máximo PPNA a fines de Octubre y un segundo pico en otoño. 
Para la zona de coironales de Magallanes (Sitio 2, área de Punta Arenas), la estación de crecimiento comienza los últimos días de Agosto y principios de Septiembre (SAG, 2004). Domínguez et al. (2019) consideran para el Distrito agroclimático de Punta Arenas, de la Región de Magallanes, una duración de 200 a 249 días de la estación de crecimiento. Según los datos de presente trabajo la duración de la estación de crecimiento, según la curva de NDVI está dentro de estos límites (aproximadamente 213 días). Para este Sitio, la alta disponibilidad de agua durante todo el año (alto NDWI), permite que el crecimiento de la vegetación ocurra en un solo momento del año (de la segunda quincena de Septiembre hasta la primera semana de Abril). Este crecimiento se ve condicionado solo por temperatura (alta correlación NDVI y temperatura).

Jobbágy, Sala y Paruelo (2002), utilizan una metodología que sólo incorpora la variable temperatura en su análisis, la cual aplicada a nuestros datos (temperatura de Julio de $2^{\circ} \mathrm{C}$ para ambos Sitios) indicaría que la estación de crecimiento sería a mediados de Septiembre, coincidiendo con lo obtenido en este trabajo para el Sitio 2, aunque desfazada en el Sitio 1 por ser este último más dependiente de la precipitación.

En relación a la duración de la estación de crecimiento ambos Sitios mostraron resultados similares, pero considerando la I-NDVI, el Sitio 2 presentaría mayor PPNA $(0,59)$ en relación al Sitio $1(0,31)$.

El comportamiento dentro del año, de la temperatura, la precipitación y los vientos, permiten explicar el comportamiento del NDWI y de la vegetación (observado con el NDVI) para el Sitio 1. El crecimiento acelerado en la primavera se debe a la disponibilidad de agua en el suelo (NDWI más alto, debido a las precipitaciones invernales) y aumento de las temperaturas. El crecimiento comienza a desacelerarse en noviembre-diciembre cuando las temperaturas son aún más altas, lo que hace que las precipitaciones estivales queden poco disponibles para las plantas (menor NDWI) debido a la evapotranspiración. El rebrote otoñal comienza porque las temperaturas son adecuadas aún para el crecimiento y existe disponibilidad de agua. El contenido de humedad del suelo (menor NDWI) disminuye en algunos momentos del año, debido a las mayores temperaturas y vientos (aumentando la evapotranspiración). Un proceso parecido ocurre cuando se produce el crecimiento de vegetación (primavera), donde las plantas comienzan a utilizar el agua del suelo, aumenta su transpiración, disminuyendo el contenido hídrico en el perfil del suelo, dependiendo adicionalmente de la profundidad de sus raíces (Paruelo y Sala, 1995).

Farías et. al (2005) describieron a la variación decadal del NDVI, en relación a distintos índices climáticos definidos en relación a la precipitación y la temperatura, e integrando información agrícola en el N-O de Argentina, así como la correlación espacial entre el índice NDVI y la PP acumulada en Marzo-Agosto. Encontraron que en algunas áreas de estudio existe una relación muy fuerte entre el desarrollo estacional de la vegetación y las precipitaciones de los meses anteriores. Por otro lado, Alatorre et al (2010) analizaron la evolución espacio temporal del índice NDVI en áreas con distinta cantidad de cobertura vegetal en dos periodos mediante modelos de regresión multivariado que incluyeron condiciones climáticas para determinar de qué manera el clima influye sobre la vegetación. Estos autores concluyen que la temperatura resultó ser el factor más explicativo, mientras que la precipitación influyó, aunque solo en un tipo de vegetación específica dentro de las analizadas. En nuestro caso, los resultados obtenidos desde los modelos planteados nos permiten concluir que en el caso del Sitio 1 (Río Gallegos), la temperatura, el balance hídrico, la precipitación del mes, así como la de los tres meses anteriores (especialmente estos últimos en el periodo Marzo-Abril), influyen en el periodo de crecimiento; mientras que en el caso del Sitio 2 (Punta Arenas) la temperatura resulta ser el factor clave sobre NDVI, y por lo tanto sobre PPNA. 
El uso complementario de datos meteorológicos y espectrales y los índices calculados a partir de ellos, permitió describir el comportamiento de la vegetación dentro del año. Sin embargo, por no contar con datos de campo de PPNA de cada Sitio, solo puede ser utilizado para conocer su variabilidad dentro del año y en el espacio analizado.

El presente trabajo es un estudio preliminar que podría enriquecerse a futuro, incorporando en su análisis, datos de temperatura superficial, humedad relativa porcentual, dirección de los vientos diferenciada, heliofanía, contenido de humedad condensada sobre el suelo, entre otros.

\section{BIBLIOGRAFÍA}

ALATORRE, L., BEGUERÍA, S. y VICENTE-SERRANO, S. (2010). Análisis de la evolución espacio-temporal del NDVI sobre áreas vegetadas y zonas de riesgo de erosión en el Pirineo central. Revista de Ecología de Montaña. Vol. 165, 7-27.

BALDI, G., VERÓN, S. R. y JOBBÁGY, E. G. (2013). The imprint of humans on landscape patterns and vegetation functioning in the dry subtropics. Glob. Change Biol. 19, 441458. https://doi.org/10.1111/gcb.12060

BORRELLI, P. (1998). Efecto de la intensidad de pastoreo sobre distintas variables del sistema suelo- planta- animal y factores limitantes de la producción ovina. Período 1990- 1997. (Informe final). INTA. EEA Santa Cruz.

CORONATO, A., MAZZONI, E., VÁZQUEZ, M. y CORONATO, F. (2017). Clima, en: Patagonia: Una Síntesis de Su Geografía Física. pp. 57-69.

COSTANZA, R., D’ARGE, R., DE GROOT, R., FARBER, S., GRASSO, M., HANNON, B., LIMBURG, K., NAEEM, S., O’NEILL, R. V., PARUELO, J., RASKIN, R. G., SUTTON, P. y VAN DEN BELT, M. (1998). The value of ecosystem services: putting the issues in perspective. Ecol. Econ. 25, 67-72. https://doi.org/10.1016/S0921$\underline{\text { 8009(98)00019-6 }}$

DI BELLA, C., REBELLA, C. y PARUELO, J. (2000). Evapotranspiration estimates using NOAA AVHRR imagery in the Pampa region of Argentina. International Remote Sensing. 21, 791-797.

DI RIENZO, J. A., CASANOVES, F., BALZARINI, M. G., GONZALEZ, L., TABLADA, M. y ROBLEDO, C.W. (2018). InfoStat versión 2018. Centro de Transferencia InfoStat, FCA, Universidad Nacional de Córdoba, Argentina. URL http://www.infostat.com.ar

DOMÍNGUEZ, E., PÉREZ, C., SUÁREZ, A., LIRA, R, IVELIC-SÁEZ, J. y DE LA FUENTE, J. (2019). Manual para estimar la cantidad de forraje disponible en pastizales naturales: una herramienta necesaria para un uso racional de estos ecosistemas. Instituto de Investigaciones Agropecuarias. Centro Regional de Investigación Kampenaike. Punta Arenas, Chile. Boletín INIA № 401. 134 pp

ENDLICHER, W. y SANTANA, A. (1988). El clima del sur de la Patagonia y sus aspectos ecológicos. Un siglo de mediciones climatológicas en Punta Arenas. Anales Instituto Patagonia Serie Cs. Nat. (Chile) 18, 57-86.

FABRICANTE, I. (2005). Variaciones Espaciales y Temporales del Índice Verde Normalizado en el Norte de la Patagonia (Tesis de Maestría). Universidad de Buenos Aires, Buenos Aires.

FARÍAS, G. P., DESSÍ, A., MASELLI, F. y CROTTI, C. (2005). Integración de series de datos satelitales y climáticos para la definición de áreas climáticamente homogéneas en el Centro-Nordeste de Argentina. Revista Geográfica Digital (IGUNNE). Vol 2 (3) Recuperado de https://revistas.unne.edu.ar/index.php/geo/article/view/2652 
FERRANTE, D. (2011). Distribución del agua en el suelo, en relación con la estructura radical y producción de biomasa de tres tipos funcionales en un pastizal de la Estepa Magallánica Seca, en Santa Cruz. (Maestría). Universidad de Buenos Aires, Buenos Aires.

GAO, B. (1996). NDWI-A normalized difference water index for remote sensing of vegetation liquid water from space. Remote Sens. Environ. 58, 257-266.

GILABERT, M. A., GONZÁLEZ- PIQUERAS, J. y GARCÍA- HARO, J. (1997). Acerca de los índices de vegetación. Rev. Teledetec. 8.

GOWARD, S. N., TUCKER, C. J. y DYE, D. G. (1985). North American vegetation patterns observed with the NOAA-7 advanced very high resolution radiometer. Vegetatio 64, 3-14. https://doi.org/10.1007/BF00033449

GU, Y., BROWN, J. F., VERDIN, J. P., y WARDLOW, B. D. (2007). A five-year analysis of MODIS NDVI and NDWI for grassland drought assessment over the central Great Plains of the United States. https://doi.org/10.1029/2006gl029127

GUTTMAN, N. B. (1994). On the sensitvity of sample L Moments to sample size. Journal of Climate, 7: 1026-1029.

GUTTMAN, N. B. (1999). Acccepting the Standardized Precipitation Index: a calculation algorithm. JAWRA Journal of the American Water Resources Association, 35(2): 311322.

HILL, M. J., DONALD, G. E., HYDER, M. W. y SMITH, R. C. G. (2004). Estimation of pasture growth rate in the south west of Western Australia from AVHRR NDVI and climate data. Remote Sens. Environ. 93, 528-545.

HUETE, A., DIDAN, K., MIURA, T., RODRIGUEZ, E. P., GAO, X. y FERREIRA, L. G. (2002). Overview of the radiometric and biophysical performance of the MODIS vegetation indices. Remote Sens. Environ. 83, 195-213.

HUNT, E. y ROCK, B. (1989). Detection of Changes in Leaf Water Content Using Near- and Middle-Infrared Reflectances. Remote Sens. Environ. 30, 43-54.

IRISARRI, G. (2008). Variación espacial y temporal de la productividad primaria neta aérea de mallines del Noroeste de la Patagonia (Tesis de Maestría). Universidad de Buenos Aires, Buenos Aires.

JI, L. y PETERS, A. (2003). Assessing vegetation response to drought in the northern Great Plains using vegetation and drought indices. Remote Sens. Environ. 87, 85-98.

JOBBÁGY, E. G., SALA, O. E. y PARUELO, J. M. (2002). Patterns and controls of primary production in the patagonian steppe: a remote sensing approach. Ecology 83, 307-319.

JÖNSSON, P. y EKLUNDH, L. (2006). TIMESAT a Program for Analyzing Time-Series of Satellite Sensor Data. Users Guide TIMESAT 23.

KADUK, J. D. y HEIMANN, M. (1996). A prognostic phenology scheme for global terrestrial carbon cycle models. Clim. Res. 6, 1-19.

LAUENROTH, W. K. (1979). Grassland primary production: North American Grasslands in perspective, in: N. R. French (Ed.), Perspectives in Grassland Ecology. Ecological Studies. Springer- Verlag, New York, USA. En Sala, O. Parton, W, J., Joyce, L.A. y Lauenroth, W.K., 1988. Primary production of the Central Grassland region of the United Status. Ecology, 69 (1): 40-45., pp. 3-24.

MCKEE, T., DOESKEN, N. y KLEIST, J. (1993). The relation of drought frequency and duration to time scales, en: Proceeding of the Eight Conference on Applied Climatology [January 17-22, 1993, Boston. USA]. Boston, American Meteorological Society, pp. 179-184.

MCKEE, T, DOESKEN, N. y KLEIST, J. (1995). Drought monitoring with multiple time scales, en: Proceeding of the Ninth Conference on Applied Climatology [January 1520, 1995, Dallas USA. TX]. Dallas, American Meteorological Society, pp. 233-236. 
MCNAUGHTON, S. J., OESTERHELD, M., FRANK, D. A. y WILliAMS, K. J. (1989). Ecosystem-level patterns of primary productivity and herbivory in terrestrial habitats. Nature 341, 142-144. https://doi.org/10.1038/341142a0

ODUM, E. P. (1971). Fundamentals of ecology, $3^{\mathrm{a}}$. ed. Saunders. Philadelphia.

OESTERHELD, M., DI BELLA, C. y KERDILES, H. (1998). Relation between NOAAAVHRR satellite data and stocking rate of rangelands. Ecol. Appl. 8, 207-212.

OLIVA, G., GONZÁLEZ, L., RIAL, P. y LIVRAGHI, E. (2001). Cap. 2. El ambiente en la Patagonia Austral, in: Borrelli, P., Oliva, G. (Eds.), Ganadería Ovina Sustentable En La Patagonia Austral. INTA, Buenos Aires.

PAREDES, P. (2011). Caracterización funcional de la Estepa Magallánica y su transición a Matorral de Mata Negra (Patagonia Austral) a partir de imágenes de resolución espacial intermedia (Tesis de Maestría). Escuela para Graduados Ing. Agr. Alberto Soriano Facultad de Agronomía - Universidad de Buenos Aires, Buenos Aires.

PARUELO, J., EPSTEIN, H. E., LAUENROTH, W. K. y BURKE, I. C. (1997). ANPP estimates from NDVI for the Central Grassland Region of the US. Ecology 78, 953958.

PARUELO, J. M., JOBBÁGY, E. G. y SALA, O. E. (1998). Biozones of Patagonian (Argentina). Ecol. Austral 8, 145-153.

PARUELO, J. M. y LAUENROTH, W. K. (1998). Interannual variability of NDVI and its relationship to climate for North American shrublands and grasslands. J. Biogeogr. 25, 721-733.

PARUELO, J. M., OESTERHELD, M., DI BELLA, C. M., ARZADUN, M., LAFONTAINE, J., CAHUEPÉ, M. y REBELLA, C. M. (2000). Estimation of primary production of subhumid rangelands from remote sensing data. Appl. Veg. Sci. 3, 189-195.

PARUELO, J. y SALA, O. (1995). Water losses in the Patagonian steppe: a modelling approach. Ecology 76, 510-520.

PICKUP, G., CHEWINGS, V. y NELSON, D. (1993). Estimating changes in vegetation cover over time in arid rangelands using Landat MSS data. Remote Sens. Environ. 43, 243-263.

R Core Team (2018). R: A language and environment for statistical computing. R Foundation for Statistical Computing, Vienna, Austria. URL https://www.R-project.org/.

REED, B. C., BROWN, J. F., VANDERZEE, D., LOVELAND, T. R., MERCHANT, J. W. y OHLEN, D. O. (1994). Measuring phenological variability from satellite imagery. J. Veg. Sci. 5, 703-714. https://doi.org/10.2307/3235884

ROUSE, W., HAAS, R. H., SCHELl, J. A. y DEERING, D. W. (1973). Monitoring vegetation Systems in the great plains with ERTS 9.

SAG, (2004). El pastizal de Magallanes, guía uso, condición actual y propuesta de seguimiento para determinación de tendencia. (eds.) Servicio Agrícola y Ganadero, XII Región de Magallanes.

SALA, O. y AUSTIN, A. (2000). Methods of estimating Aboveground Primary Production. O.Sala- R. Jackson- H. Mooney \& R. Howarth editores, in: Methods in Ecosystem Science. New York Springer Verlag.

SALA, O. E., LAUENROTH, W. K. y GOLLUSCIO, R. A. (1997). Plant functional types in temperate semi-arid regions, in: Smith, T.M., Shugart, H.H., Woodward, F.I. (Eds.), Plant Functional Types - Their Relevance to Ecosystem and Global Change. Cambridge Universtity Press, Cambridge, pp. 217-233.

SALA, O. E., PARTON, W. J., JOYCE, L. A. y LAUENROTH, W. K. (1988). Primary production of the Central Grassland Region of the United States. Ecology 69, 40-45. 
SOlBRIG, O. T., BARBOUR, M. A., CROSS, J., GOLDSTEIN, G., LOWE, C. H., MORELLO, J. y YOUNG, T. W. (1977). The strategies and community patters of desert plants, in: Orians G. H. \& Solbrig, O.T. (Ed.), Convergent Evolution in Warm Desert. Downden, Hutchinson and Ross, Inc. Stroudsburg, PA. Citado en Golluscio, R. \& Sala, O. (1993) Plant functional types and ecological strategies in Patagonian forbs. Journal of Vegetation Science, 4, 839- 46., pp. 67-106.

THORNTHWAITE, C. W. (1948). An approach toward a rational classification of climate. Geographical Review 38: 55-94. https://doi.org/10.1097/00010694-194807000-00007

VICENTE-SERRANO, S. M., BEGUERÍA, S. y LÓPEZ-MORENO, J. (2010). A Multiscalar drought index sensitive to global warming: The Standardized Precipitation Evapotranspiration Index - SPEI. Journal of Climate 23:1696-1718

VOLANTE, J. N., ALCARAZ-SEGURA, D., MOSCIARO, M. J., VIGLIZZO, E. F. y PARUELO, J. M. (2012). Ecosystem functional changes associated with land clearing in NW Argentina. Agric. Ecosyst. Environ. 154, 12-22. https://doi.org/10.1016/j.agee.2011.08.012

WAN, Z. (1999). MODIS Land-Surface Temperature. Algorithm theoretical basis document (LST ATBD). Version 3.3. Inst. Comput. Earth Syst. Sci. Univ. Calif. St. Barbara.

WHITE, M., THORNTON, P. y RUNNING, S. (1997). A continental phenology model for monitoring vegetation responses to interannual climatic variability. Glob. Biogeochem. Cycles 11, 217-234. 